\title{
Stability and stabilization of ecosystem for epidemic virus transmission under Neumann boundary value via impulse control
}

\author{
Ruofeng Rao ${ }^{\mathrm{a}}$ \\ ${ }^{a}$ Department of Mathematics, Chengdu Normal University, Chengdu , 611130, China
}

\begin{abstract}
In this paper, by using the variational method, a sufficient condition for the unique existence of the stationary solution of the reaction-diffusion ecosystem is obtained, which directly leads to the global asymptotic stability of the unique equilibrium point. Besides, employing impulse control technique derives the globally exponential stability criterion of delayed feedback ecosystem. And numerical examples illuminate the effectiveness of impulse control, which has a certain enlightening effect on the actual epidemic prevention work. That is, in the face of the epidemic situation, taking a certain frequency of positive and effective epidemic prevention measures is conducive to the stability and control of the epidemic situation. particularly, the newly-obtained theorems quantifies this feasible step.
\end{abstract}

Keywords: Neumann boundary value, positive equilibrium point, Poincare inequality lemma, impulse control

\section{Introduction}

Infectious diseases have existed for thousands of years in the history of human development and brought great harm to human beings. In the 6th century, the prevalence of smallpox in the Middle East reduced the population by nearly one tenth. From 1347 to 1352, the spread of plague in Europe killed nearly one third of the European population. AIDS is due to the infection of human immunodeficiency virus and make the human immune system destroyed, loss of immune capacity. In 1927, Kermack and McKendrick established the famous Sir chamber model in order to analyze the spreading law of London black death from 1665 to 1666 and Bombay plague in 1906 ([3]). In 1932, Kermack and McKendrick established the famous SIS model ([4,5]). The idea of compartment model is to divide the population into susceptible individuals, infected individuals and recovering individuals, and ignore the birth and death of the population, that is, the total population remains unchanged. Such infectious diseases as measles and chickenpox, patients with lifelong immunity after recovery, recovery individuals will not enter the susceptible individuals. On this basis, numerous scholars have done a lot of work ([6-10]). Stability analysis is an important part of infectious disease dynamics. The stability of equilibrium point is studied by qualitative theory of delay differential equations and uniform persistence theory. The stability of equilibrium includes local stability, uniform persistence and global stability. Local stability means that the initial value is near the equilibrium point, and the value at any

Email address: ruofengrao@163.com (Ruofeng Rao )

Preprint submitted to $* * *$

February 6, 2021 
time is near the equilibrium point [6]. Uniform persistence means that the system eventually has a positive lower bound, which reveals that infectious diseases eventually spread in the population [7]. Global stability means that the equilibrium point is stable. For any initial value, when the time is sufficiently large, the solution of the system will converge to the equilibrium point [7,8], The disease is extinct or epidemic. The research on the stability and consistent persistence of the dynamic model of infectious diseases is helpful for people to find the epidemic law of infectious diseases, and is of great significance for the prevention and control of infectious diseases. Time delay effect exists widely in the objective world. The epidemic law of infectious diseases not only depends on the current state, but also related to the previous state. In the process of infectious disease transmission, for some infectious diseases, such as tuberculosis, AIDS, etc., the susceptible become infected after infection, but they do not necessarily have the ability to infect immediately. It takes a period of time for the susceptible to become infected to have the ability to infect, so people need to take the infection delay into account in the infectious disease model. For influenza and other diseases, since the infected person can still reinfect the disease after recovery, it is necessary to consider the recovery delay. The recovery delay is the time from the recovery stage of the convalescent person to becoming a susceptible person again. In order to study gonorrhea, Cooke and Yorke established an epidemic dynamics model with time delay ([9]). There are a lot of classical works on the time-delay epidemic model (see [10 - 13]). In the virus dynamics model, since it takes a period of time from the virus infecting the host cell to the host cell producing virus particles, it is necessary to take the infection delay into account in the virus dynamics model. Because there is a period of time between the immune system receiving antigen stimulation and producing immune cells, it is necessary to take the immune delay into account in the virus dynamics model. In this paper, a dynamic model of infectious disease patients and susceptible patients with diffusion is studied, and a global stability result is obtained. Furthermore, since the artificial epidemic prevention can be regarded as an impulsive effect, the impulsive delay dynamical system is also considered, and the global exponential asymptotic stability criterion is obtained.

In this paper, the innovative points are listed as follows,

$\diamond$ By using the variational method, a sufficient condition for the unique existence of the stationary solution of the reaction-diffusion ecosystem is obtained, which directly leads to the global asymptotic stability of the unique equilibrium point.

$\diamond$ Utilizing impulse control technique derives the globally exponential stability criterion of delayed feedback ecosystem. And numerical examples illuminate the effectiveness of impulse control, which has a certain enlightening effect on the actual epidemic prevention work .

For convenience, throughout of this paper, the author denotes by $\lambda_{1}$ the smallest positive eigenvalue of the Neumann boundary problem in [1, Lemma 4]. Symmetric matrix $A>0$ represents $A$ is a positive definite symmetric matrix, and $A>B$ or $B<A$ represents $A-B$ is a positive definite symmetric matrix. $\lambda_{\max } A$ represents the maximum eigenvalue of the symmetric matrix $A$. For a matrix $A=\left(a_{i j}\right)_{n \times n}$, denote $|A|=\left(\left|a_{i j}\right|\right)_{n \times n}$. For a vector $U=\left(U_{1}, U_{2}\right)^{T}$, denote $|U|=\left(\left|U_{1}\right|,\left|U_{2}\right|\right)^{T}$. 


\section{System descriptions}

Consider the following ecosystem under Neumann boundary value:

$$
\left\{\begin{array}{l}
\frac{\partial u}{\partial t}=q_{1} \Delta u+a_{1} u v-b_{1} u v^{2}-c_{1} u, \quad t \geqslant 0, x \in \Omega, \\
\frac{\partial v}{\partial t}=q_{2} \Delta v-a_{2} u v+b_{2} u^{2} v-c_{2} u+d, \quad t \geqslant 0, x \in \Omega, \\
\frac{\partial u}{\partial v}=0=\frac{\partial v}{\partial v}, \quad x \in \partial \Omega, t \geqslant 0 .
\end{array}\right.
$$

where $u, v$ represent population density of people infected with virus and that of virus susceptible people or animal, respectively. $d>0$ represents the birth rate of the virus susceptible, $a_{1} u v$ represents the increasing degree on the infected with virus due to the cross infection, and $-a_{2} u v$ represents the decreasing degree on the susceptible due to the cross infection. $b_{1} u v^{2}$ represents decreasing degree on the infected due to epidemic preventions while $b_{2} u^{2} v$ is the increasing degree on the susceptible after the patients are cured and became susceptible to infection. $c_{1} u$ represents the decreasing degree on the patients due to the death of some patients. $c_{2} u$ represents the decreasing degree on the virus susceptible due to increasing patients.

It is well known that if $(u, v)$ is a constant equilibrium point of the system (2.1), then

$$
\left\{\begin{array}{l}
a_{1} u v-b_{1} u v^{2}-c_{1} u=0, \\
-a_{2} u v+b_{2} u^{2} v-c_{2} u+d=0,
\end{array}\right.
$$

Throughout this paper, the following assumption is considered,

(H1) There are two positive constants $M_{i}(i=1,2)$ such that

$$
0 \leqslant u \leqslant M_{1}, \quad 0 \leqslant v \leqslant M_{2}
$$

(H2) Assume that the positive numbers $a_{i}, b_{i}, c_{i}, d$ satisfy

$$
\left\{\begin{array}{l}
a_{1}^{2}=4 b_{1} c_{1} \\
\left(a_{1} a_{2}+2 b_{1} c_{2}\right)^{2}=8 a_{1} b_{1} b_{2} d
\end{array}\right.
$$

Remark 1. Due to the limited natural resources, the boundedness hypothesis (H1) on population density is reasonable.

Remark 2. For any given positive numbers $a_{i}, b_{i}, c_{i}, d$ satisfying (2.4), the condition (H2) can guarantee that the positive solution $\left(u_{*}, v_{*}\right)^{T}$ of the equations (2.2) is unique, where

$$
u_{*}=\frac{a_{1} a_{2}+2 b_{1} c_{2}}{2 a_{1} b_{2}}, \quad v_{*}=\frac{a_{1}}{2 b_{1}} .
$$

But the condition (H2) can not warrant that $\left(u_{*}, v_{*}\right)^{T}$ must be the unique stationary solution of the system (2.1). In fact, each $(u(x), v(x))^{T}$ satisfying the following elliptic equations (2.6) must be a stationary solution of the ecosystem (2.1), where 


$$
\left\{\begin{array}{l}
q_{1} \Delta u+a_{1} u v-b_{1} u v^{2}-c_{1} u=0, \quad x \in \Omega, \\
q_{2} \Delta v-a_{2} u v+b_{2} u^{2} v-c_{2} u+d=0, \quad x \in \Omega, \\
\frac{\partial u}{\partial v}=0=\frac{\partial v}{\partial v}, \quad x \in \partial \Omega .
\end{array}\right.
$$

The following lemma may be necessary to some extent (see, e.g. [1, Lemma 4]).

Lemma 2.1.(Poincar Integral Inequality). Let $\Omega$ be a bounded domain of $R^{m}$ with a smooth boundary $\partial \Omega$ of class $C^{2}$ by $\Omega . v(x)$ is a real-valued function belonging to $H_{0}^{1}(\Omega)$ and $\left.\frac{\partial v(x)}{\partial v}\right|_{\partial \Omega}=0$. Then

$$
\lambda_{1} \int_{\Omega}|v(x)|^{2} d x \leqslant \int_{\Omega}|\nabla v(x)|^{2} d x,
$$

which $\lambda_{1}$ is the smallest positive eigenvalue of the Neumann boundary problem

$$
\begin{cases}-\Delta \varphi=\lambda \varphi, & x \in \Omega \\ \frac{\partial \varphi(x)}{\partial v}=0, & x \in \partial \Omega .\end{cases}
$$

\section{Unique existence of the globally asymptotical stationary solution}

Due to the viewpoint of Remark 2, the author has to prove that $\left(u_{*}, v_{*}\right)^{T}$ defined in $(2.5)$ is the unique equilibrium point of the system (2.1). The following uniqueness proof technique imitates that of the author's another work [14].

Theorem 3.1. Suppose the conditions (H1) and (H2) hold, and

$$
\left(\begin{array}{cc}
\alpha_{1} & \frac{1}{2}\left(\alpha_{2}+\beta_{1}\right) \\
* & \beta_{2}
\end{array}\right)<\lambda_{1} Q,
$$

then $\left(u_{*}, v_{*}\right)^{T}$ defined in (2.5) is the unique stationary solution of the ecosystem (2.1), where $\lambda_{1}$ is the smallest positive eigenvalue of the Neumann boundary problem ([1, Lemma 4]), and

$$
\begin{aligned}
& \alpha_{1}=\left|a_{1} v_{*}-b_{1} v_{*}^{2}-c_{1}\right|+\left|a_{1}-2 b_{1} v_{*}\right| \cdot M_{2}, \quad \beta_{1}=\left|a_{1} u_{*}-2 b_{1} u_{*} v_{*}\right|+b_{1} u_{*} M_{2}+b_{1} M_{1} M_{2}, \\
& \alpha_{2}=\left|-a_{2} v_{*}+2 b_{2} u_{*} v_{*}-c_{2}\right|+b_{2} v_{*} M_{1}+b_{2} M_{1} M_{2}, \quad \beta_{2}=\left|-a_{2} u_{*}+b_{2} u_{*}^{2}\right|+\left|-a_{2}+2 b_{2} u_{*}\right| \cdot M_{1} .
\end{aligned}
$$

Proof. It is easy to verify that $\left(u_{*}, v_{*}\right)$ defined in (2.5) is a solution of the following equations

$$
\left\{\begin{array}{l}
a_{1} u v-b_{1} u v^{2}-c_{1} u=0, \\
-a_{2} u v+b_{2} u^{2} v-c_{2} u+d=0,
\end{array}\right.
$$

which implies that $\left(u_{*}, v_{*}\right)^{T}$ is a positive equilibrium point of the system (2.1).

Let $(u(x), v(x))^{T}$ be any stationary solution of the system (2.1). Set $z_{1}=u(x)-u_{*}, z_{2}=v(x)-v_{*}$, and $z=\left(z_{1}, z_{2}\right)^{T}$, then 


$$
\left\{\begin{array}{l}
q_{1} \Delta z_{1}+\phi_{1}\left(z_{1}, z_{2}\right)=0, \quad x \in \Omega, \\
q_{2} \Delta z_{2}+\phi_{2}\left(z_{1}, z_{2}\right)=0, \quad x \in \Omega, \\
\frac{\partial z_{1}}{\partial v}=0=\frac{\partial z_{2}}{\partial v}, \quad x \in \partial \Omega,
\end{array}\right.
$$

where

$$
\left\{\begin{array}{l}
\phi_{1}(z)=\left(a_{1} v_{*}-b_{1} v_{*}^{2}-c_{1}\right) z_{1}+\left(a_{1} u_{*}-2 b_{1} u_{*} v_{*}\right) z_{2}+\left(a_{1}-2 b_{1} v_{*}\right) z_{1} z_{2}-b_{1} u_{*} z_{2}^{2}-b_{1} z_{1} z_{2}^{2}, \\
\phi_{2}(z)=\left(-a_{2} v_{*}+2 b_{2} u_{*} v_{*}-c_{2}\right) z_{1}+\left(-a_{2} u_{*}+b_{2} u_{*}^{2}\right) z_{2}+\left(-a_{2}+2 b_{2} u_{*}\right) z_{1} z_{2}+b_{2} v_{*} z_{1}^{2}+b_{2} z_{1}^{2} z_{2},
\end{array}\right.
$$

Based on the boundedness assumptions (H1) on $u, v$, one can get

$$
\left|\phi_{1}\left(z_{1}, z_{2}\right)\right| \leqslant \alpha_{1}\left|z_{1}\right|+\beta_{1}\left|z_{2}\right|
$$

and

$$
\left|\phi_{2}\left(z_{1}, z_{2}\right)\right| \leqslant \alpha_{2}\left|z_{1}\right|+\beta_{2}\left|z_{2}\right|
$$

where $\alpha_{i}, \beta_{i}$ are defined in (3.2).

Similarly as that of [1], employing Poincare integral inequality ([1, Lemma 4]) results in

$$
\lambda_{1} \int_{\Omega}|z|^{T} Q|z| \leqslant \int_{\Omega}\left(\alpha_{1}\left|z_{1}\right|^{2}+\left(\alpha_{2}+\beta_{1}\right)\left|z_{1} z_{2}\right|+\beta_{2}\left|z_{2}\right|^{2}\right)
$$

or

$$
\lambda_{1} \int_{\Omega}|z|^{T} Q|z| \leqslant \int_{\Omega}|z|^{T}\left(\begin{array}{cc}
\alpha_{1} & \frac{1}{2}\left(\alpha_{2}+\beta_{1}\right) \\
* & \beta_{2}
\end{array}\right)|z|,
$$

and hence $z=0$, and $u(x)=u_{*}, v(x)=v_{*}$. The proof of Theorem 3.1 is completed.

Remark 3. Both $u_{*}$ and $v_{*}$ are only the positive numbers, dependent upon $a_{i}, b_{i}, c_{i}$ and $d$. Particularly, $a_{1} v_{*}-b_{1} v_{*}^{2}-c_{1}=$ 0 and so $\alpha_{1}=\left|a_{1} v_{*}-b_{1} v_{*}^{2}-c_{1}\right|+\left|a_{1}-2 b_{1} v_{*}\right| \cdot M_{2}$. Similarly, other parameters are also simplified to some extent.

Set $U=U(t, x)=\left(U_{1}(t, x), U_{2}(t, x)\right)^{T}$ with

$$
\left\{\begin{array}{c}
U_{1}(t, x)=u(t, x)-u_{*} \\
U_{2}(t, x)=v(t, x)-v_{*}
\end{array}\right.
$$

where $u_{*}$ and $v_{*}$ are positive numbers, defined in (2.5). Then it follows from (2.1) that

$$
\begin{cases}\frac{\partial\left(u-u_{*}\right)}{\partial t}=q_{1} \Delta\left(u-u_{*}\right)+a_{1} u v-a_{1} u_{*} v_{*}-b_{1} u v^{2}+b_{1} u_{*} v_{*}-c_{1}\left(u-u_{*}\right), & t \geqslant 0, x \in \Omega, \\ \frac{\partial\left(v-v_{*}\right)}{\partial t}=q_{2} \Delta\left(v-v_{*}\right)-a_{2} u v+a_{2} u_{*} v_{*}+b_{2} u^{2} v-b_{2} u_{*}^{2} v_{*}-c_{2}\left(u-u_{*}\right), \quad t \geqslant 0, x \in \Omega, & \\ \frac{\partial\left(u-u_{*}\right)}{\partial v}=0=\frac{\partial\left(v-v_{*}\right)}{\partial v}, & x \in \partial \Omega, t \geqslant 0\end{cases}
$$


which can be rewritten as follows,

$$
\left\{\begin{array}{l}
\frac{\partial U}{\partial t}=Q \Delta U+\phi(U), \quad t \geqslant 0, x \in \Omega \\
\frac{\partial U}{\partial v}=0, \quad x \in \partial \Omega, t \geqslant 0
\end{array}\right.
$$

where $\phi(U)=\left(\phi_{1}(U), \phi_{2}(U)\right)^{T}$, and (3.4) yields

$$
\left\{\begin{array}{l}
\phi_{1}(U)=\left(a_{1} v_{*}-b_{1} v_{*}^{2}-c_{1}\right) U_{1}+\left(a_{1} u_{*}-2 b_{1} u_{*} v_{*}\right) U_{2}+\left(a_{1}-2 b_{1} v_{*}\right) U_{1} U_{2}-b_{1} u_{*} U_{2}^{2}-b_{1} U_{1} U_{2}^{2}, \\
\phi_{2}(U)=\left(-a_{2} v_{*}+2 b_{2} u_{*} v_{*}-c_{2}\right) U_{1}+\left(-a_{2} u_{*}+b_{2} u_{*}^{2}\right) U_{2}+\left(-a_{2}+2 b_{2} u_{*}\right) U_{1} U_{2}+b_{2} v_{*} U_{1}^{2}+b_{2} U_{1}^{2} U_{2} .
\end{array}\right.
$$

Obviously, the positive equilibrium point $\left(u_{*}, v_{*}\right)^{T}$ of the system $(2.1)$ is corresponding to the null solution $(0,0)^{T}$ of the system (3.8), where $u_{*}$ and $v_{*}$ are positive numbers, defined in (2.5).

Theorem 3.2. If all the conditions of Theorem 3.1 are satisfied, then the unique equilibrium point $\left(u_{*}, v_{*}\right)^{T}$ of the system (2.1) is globally asymptotically stable.

Proof. Consider the Lyapunov functional as follows

$$
V(t, U)=\int_{\Omega} U^{T}(t, x) U(t, x) d x .
$$

It is follows from (3.5) and (3.6) that

$$
\left|\phi_{1}(U)\right| \leqslant \alpha_{1}\left|U_{1}\right|+\beta_{1}\left|U_{2}\right|
$$

and

$$
\left|\phi_{2}(U)\right| \leqslant \alpha_{2}\left|U_{1}\right|+\beta_{2}\left|U_{2}\right|,
$$

Combining (3.11) and (3.12) results in

$$
\begin{aligned}
\left|\phi(U)^{T} U\right| & =\left|U^{T} \phi(U)\right|=\left|U_{1} \phi_{1}(U)+U_{2} \phi_{2}(U)\right| \\
& \leqslant|U|^{T}\left(\begin{array}{cc}
\alpha_{1} & \frac{1}{2}\left(\alpha_{2}+\beta_{1}\right) \\
* & \beta_{2}
\end{array}\right)|U|
\end{aligned}
$$

Then Poincare inequality Lemma ([1, Lemma 4]), (3.1) and (3.8) yield

$$
\begin{aligned}
\frac{d U}{d t} & =2 \int_{\Omega} U^{T}(Q \Delta U+\phi(U)) d x \\
& \leqslant 2 \int_{\Omega}\left[-\lambda_{1}|U|^{T} Q|U|+|U|^{T}\left(\begin{array}{cc}
\alpha_{1} & \frac{1}{2}\left(\alpha_{2}+\beta_{1}\right) \\
* & \beta_{2}
\end{array}\right)|U|\right] \leqslant 0 .
\end{aligned}
$$

Besides,

$$
\|U\|_{L^{2}(\Omega)}^{2} \leqslant V(t, u) \leqslant 2\|U\|_{L^{2}(\Omega)}^{2}
$$

which together with (3.14) implies that the null solution of the system (3.8) is globally asymptotically stable, and hence, the unique equilibrium point $\left(u_{*}, v_{*}\right)^{T}$ of the system $(2.1)$ is globally asymptotically stable. 


\section{Impulse control and global stabilization on delayed feedback system}

Consider the following delayed feedback system with impulse:

$$
\left\{\begin{aligned}
\frac{\partial U}{\partial t} & =Q \Delta U+\phi(U)+P(U(t, x)-U(t-\tau(t), x)), \quad t \geqslant 0, t \neq t_{k}, x \in \Omega, \\
U\left(t_{k}^{+}, x\right) & =B_{k} U\left(t_{k}^{-}, x\right), \quad k=1,2, \cdots \\
\frac{\partial U}{\partial v} & =0, \quad x \in \partial \Omega, t \geqslant 0, \\
U(s, x) & =\xi(s, x), \quad s \in[-\tau, 0], x \in \Omega,
\end{aligned}\right.
$$

where $U$ is defined in (3.7), $\tau(t) \in[-\tau, 0], U\left(t_{k}^{+}, x\right)=U\left(t_{k}, x\right)$ and

$$
P=\left(\begin{array}{cc}
p_{1} & 0 \\
* & p_{2}
\end{array}\right)
$$

Obviously, the null solution of (4.1) is corresponding to the equilibrium point $\left(u_{*}, v_{*}\right)^{T}$ of the following system:

$$
\left\{\begin{array}{l}
\frac{\partial u}{\partial t}=q_{1} \Delta u+a_{1} u v-b_{1} u v^{2}-c_{1} u+p_{1}(u(t, x)-u(t-\tau(t), x)), \quad t \geqslant 0, t \neq t_{k} x \in \Omega, \\
\frac{\partial v}{\partial t}=q_{2} \Delta v-a_{2} u v+b_{2} u^{2} v-c_{2} u+d+p_{2}(v(t, x)-v(t-\tau(t), x)), \quad t \geqslant 0, t \neq t_{k} x \in \Omega, \\
\left(u\left(t_{k}^{+}, x\right)-u_{*}, v\left(t_{k}^{+}, x\right)-v_{*}\right)^{T}=B_{k}\left(u\left(t_{k}^{-}, x\right)-u_{*}, v\left(t_{k}^{-}, x\right)-v_{*}\right)^{T}, \quad k=1,2, \cdots \\
\frac{\partial u}{\partial v}=0=\frac{\partial v}{\partial v}, \quad x \in \partial \Omega, t \geqslant 0, \\
\left(u(s, x)-u_{*}, v(s, x)-v_{*}\right)=\xi(s, x), \quad s \in[-\tau, 0], x \in \Omega,
\end{array}\right.
$$

where each $B_{k}>0$ is a symmetric matrix, dependent on $k$.

Remark 4. Suppose all the conditions of Theorem 3.1, then $\left(u_{*}, v_{*}\right)^{T}$ defined in $(2.5)$ is the unique equilibrium point of the system (2.1), and is obviouly the unique one of the impulsive system (4.3), too. And so the unique equilibrium point $\left(u_{*}, v_{*}\right)^{T}$ of the system (4.3) is corresponding to the null solution of the system (4.1).

Based on the viewpoint of Remark 4, a stabilization criterion is presented as follows,

Theorem 4.1. Suppose all the conditions of Theorem 3.1 hold. If, in addition, there exist several positive constants $c, \lambda, \sigma$ with $\sigma-\lambda \geqslant c$, and $\gamma \geqslant 1$ such that

$$
\begin{gathered}
0<\sup _{k \in \mathbb{N}} \lambda_{\max } B_{k}^{2}<1 \\
\gamma \geqslant \frac{1}{\sup _{k \in \mathbb{N}} \lambda_{\max } B_{k}^{2}} \\
\ln \left(\sup _{k \in \mathbb{N}} \lambda_{\max } B_{k}^{2}\right)<-(\sigma-\lambda)\left(t_{k}-t_{k-1}\right), k \in \mathbb{N},
\end{gathered}
$$


then the unique equilibrium point $\left(u_{*}, v_{*}\right)^{T}$ of the system (4.3) is globally exponentially stable with convergence rate $\frac{\lambda}{2}$, where $I$ represents identity matrix, and

$$
c=\lambda_{\max }\left[-2 \lambda_{1} Q+3 P+2\left(\begin{array}{cc}
\alpha_{1} & \frac{1}{2}\left(\alpha_{2}+\beta_{1}\right) \\
* & \beta_{2}
\end{array}\right)+\gamma e^{\lambda \tau}\left(\lambda_{\max } P\right) I\right]>0 .
$$

Proof. Firstly, according to Remark 4, the conditions of Theorem 3.1 warrant that $\left(u_{*}, v_{*}\right)^{T}$ is the unique equilibrium point of the system (4.3).

Consider the Lyapunov functional as follows

$$
V(t, U)=\int_{\Omega} U^{T}(t, x) U(t, x) d x=\int_{\Omega}|U(t, x)|^{T}|U(t, x)| d x .
$$

Then Poincare inequality Lemma ([1, Lemma 4]) and (4.1) yield that for $t \in\left[t_{k-1}, t_{k}\right), t \in \mathbb{N}$,

$$
\begin{aligned}
D^{+} V(t, U(t)) & =2 \int_{\Omega} U^{T}(Q \Delta U+\phi(U)+P(U(t, x)-U(t-\tau(t), x))) d x \\
& \left.\left.\leqslant \int_{\Omega}|U|^{T}\left[-2 \lambda_{1} Q+3 P+2\left(\begin{array}{cc}
\alpha_{1} & \frac{1}{2}\left(\alpha_{2}+\beta_{1}\right) \\
* & \beta_{2}
\end{array}\right)\right]|U| d x+\lambda_{\max } P \int_{\Omega} \mid U(t-\tau(t), x)\right)\left.\right|^{T} \mid U(t-\tau(t), x)\right) \mid d x
\end{aligned}
$$

According to [2, Theorem 2.1], when

$$
\left.\left.\int_{\Omega} \mid U(t-\tau(t), x)\right)\left.\right|^{T} \mid U(t-\tau(t), x)\right)\left.\left|d x \leqslant \gamma e^{\lambda \tau} \int_{\Omega}\right| U\right|^{T}|U| d x,
$$

(4.8) yields

$$
\begin{aligned}
D^{+} V(t, U(t)) & \left.\left.\leqslant \int_{\Omega}|U|^{T}\left[-2 \lambda_{1} Q+3 P+2\left(\begin{array}{cc}
\alpha_{1} & \frac{1}{2}\left(\alpha_{2}+\beta_{1}\right) \\
* & \beta_{2}
\end{array}\right)\right]|U| d x+\lambda_{\max } P \int_{\Omega} \mid U(t-\tau(t), x)\right)\left.\right|^{T} \mid U(t-\tau(t), x)\right) \mid d x \\
& \leqslant c V(t, U(t)), \quad t \in\left[t_{k-1}, t_{k}\right), t \in \mathbb{N},
\end{aligned}
$$

where $c>0$ is defined in (4.7).

On the other hand,

$$
V\left(t_{k}, U\left(t_{k}\right)\right)=\int_{\Omega} U\left(t_{k}^{-}, x\right)^{T} B_{k} B_{k} U\left(t_{k}^{-}, x\right) d x \leqslant \lambda_{\max } B_{k}^{2} V\left(t_{k}^{-}, U\left(t_{k}^{-}\right)\right),
$$

which together with (4.9), and (4.4)-(4.6) means that all the conditions of [1, Theorem 2.1] are satisfied. According to [2, Theorem 2.1], the null solution of the system (4.1) is globally exponentially stable with convergence rate $\frac{\lambda}{2}$. And hence, the unique equilibrium point $\left(u_{*}, v_{*}\right)^{T}$ of the system (4.3) is globally exponentially stable with convergence rate $\frac{\lambda}{2}$. 


\section{Numerical examples}

\section{Example 5.1. Set}

$$
M_{1}=M_{2}=a_{2}=b_{1}=b_{2}=c_{1}=c_{2}=d=1, a_{1}=2,
$$

which means that the conditions (H1) and (H2) hold, and direct computation yields

$$
u_{*}=1=v_{*}, \alpha_{1}=0, \alpha_{2}=2=\beta_{1}, \beta_{2}=1 .
$$

Particularly, $u_{*}=1=v_{*}$ doesn't contradict the boundedness condition (H1). Moreover, set

$$
Q=\left(\begin{array}{cc}
0.25 & 0 \\
* & 0.35
\end{array}\right)
$$

and

$$
\Omega=[0,1] \times[0,1],
$$

then [1, Remark 1] tells that $\lambda_{1}=\pi^{2}$. And one can know from direct computation that the condition (3.1) holds.

According to Theorem 3.1, $(1,1)^{T}$ is the unique stationary solution of the ecosystem $(2.1)$. Moreover, Theorem 3.2 tells that the unique stationary solution $(1,1)^{T}$ of the ecosystem $(2.1)$ is globally asymptotically stable.

Example 5.2. Suppose (5.1)-(5.4) hold, and then $\lambda_{1}=\pi^{2}$. Set

$$
P=\left(\begin{array}{cc}
0.2 & 0 \\
* & 0.3
\end{array}\right), \quad B_{k} \equiv\left(\begin{array}{cc}
0.5 & 0 \\
* & 0.6
\end{array}\right), \quad k \in \mathbb{N} .
$$

And hence $\sup _{k \in \mathbb{N}} \lambda_{\max } B_{k}^{2}=0.36$. Let $\gamma=3$, and then the condition (4.5) holds.

$$
c=\lambda_{\max }\left[-2 \lambda_{1} Q+3 P+2\left(\begin{array}{cc}
\alpha_{1} & \frac{1}{2}\left(\alpha_{2}+\beta_{1}\right) \\
* & \beta_{2}
\end{array}\right)+\gamma e^{\lambda \tau}\left(\lambda_{\max } P\right) I\right]
$$

Then the condition (4.4) holds. Let

$$
\lambda=0.5, \quad t_{k}-t_{k-1} \equiv 0.5, k \in \mathbb{N}
$$

Direct computation yields

$$
c=\lambda_{\max }\left[-2 \lambda_{1} Q+3 P+2\left(\begin{array}{cc}
\alpha_{1} & \frac{1}{2}\left(\alpha_{2}+\beta_{1}\right) \\
* & \beta_{2}
\end{array}\right)+\gamma e^{\lambda \tau} \lambda_{\max } P\right]=0.9872>0 .
$$

Set $\sigma=1.5$, and then $\sigma-\lambda=1>c$. Moreover,

$$
\ln \left(\sup _{k \in \mathbb{N}} \lambda_{\max } B_{k}^{2}\right)=-1.0217<-0.5000=-(\sigma-\lambda)\left(t_{k}-t_{k-1}\right), k \in \mathbb{N}
$$


and the condition (4.6) is satisfied. Now all the conditions of Theorem 4.1 are satisfied, and Theorem 4.1 yields that the unique equilibrium point $(1,1)^{T}$ of the system $(4.3)$ is globally exponentially stable with convergence rate $25 \%$.

On the other hand, if setting $B_{k} \equiv \operatorname{diag}(0.1,0.2)$ in Example 5.2, direct computation yields that $\frac{\lambda}{2}=35 \%$; if letting $t_{k}-t_{k-1} \equiv 0.8$, direct computation yields that $\frac{\lambda}{2}=15 \%$. Such comparisons are list as follows,

Table 1.Comparisons the influences on the convergence rate $\frac{\lambda}{2}$ under different pulse amplitude with the same other data

\begin{tabular}{|c|c|c|}
\hline & Case 1 & Case 2 \\
\hline Pulse amplitude & $B_{k} \equiv \operatorname{diag}(0.5,0.6)$ & $B_{k} \equiv \operatorname{diag}(0.1,0.2)$ \\
\hline Pulse intensity & smaller & bigger \\
\hline Pulse interval & $\left(t_{k+1}-t_{k}\right) \equiv 0.5$ & $\left(t_{k+1}-t_{k}\right) \equiv 0.5$ \\
\hline Pulse frequency & same & same \\
\hline Convergence rate $\frac{\lambda}{2}$ & $25 \%$ & $35 \%$ \\
\hline
\end{tabular}

Table 2.Comparisons the influences on the convergence rate $\frac{\lambda}{2}$ under different pulse frequency with the same other data

\begin{tabular}{|c|c|c|}
\hline & Case 1 & Case 2 \\
\hline Pulse amplitude & $B_{k} \equiv \operatorname{diag}(0.5,0.6)$ & $B_{k} \equiv \operatorname{diag}(0.5,0.6)$ \\
\hline Pulse intensity & same & same \\
\hline Pulse interval & $\left(t_{k+1}-t_{k}\right) \equiv 0.5$ & $\left(t_{k+1}-t_{k}\right) \equiv 0.8$ \\
\hline Pulse frequency & smaller & bigger \\
\hline Convergence rate $\frac{\lambda}{2}$ & $25 \%$ & $15 \%$ \\
\hline
\end{tabular}

Remark 5. Table 1 illuminates that the greater the pulse intensity, the faster the stability of the system. And Table 2 indicates that the more frequent the pulses, the faster the stability of the system.

\section{Conclusions}

At first, the author uses variational methods to derive the uniqueness of the equilibrium point of ecosystem so that the global stability can be consider. After giving two global stability criteria, the author proposes numerical examples to illuminate the effectiveness of the theorems. Numerical examples and stability criteria tell that in the face of the epidemic situation, taking a certain frequency of positive and effective epidemic prevention measures is conducive to the stability and control of the epidemic situation.

\section{References}

[1] Jie Pan, Xinzhi Liu, Shouming Zhong. Stability criteria for impulsive reaction-diffusion Cohen-Grossberg neural networks with timevarying delays. Mathematical and Computer Modelling, 51 (2010) 1037-1050.

[2] Quanjun Wu, Jin Zhou, Lan Xiang. Global exponential stability of impulsive differential equations with any time delays. Applied Mathematics Letters 23 (2010) 143-147. 
[3] Kermack W O, Mc Kendrick A G. Contributions to the Mathematical Theory of Epi- demics. Proceedings of the Royal Society of London. Series A, 1927, 115(772): 700-721.

[4] Kermack W O, Mc Kendrick A G. Contributions to the Mathematical Theory of Epidemics. Proceedings of the Royal Society of London. Series A, 1932, 138(834):55-83.

[5] Kermack W O, Mc Kendrick A G. Contributions to the Mathematical Theory of Epidemics[J]. Proceedings of the Royal Society of London. Series A, 1933, 141(843):94-122.

[6] Hethcote H W, Thieme H R. Stability of the Endemic Equilibrium in Epidemic Mod- els with Subpopulations[J]. Mathematical Biosciences, 1985, 75(2):205-227.

[7] Thieme H R. Persistence under Relaxed Point-Dissipativity (with Application to an Endemic Model)[J]. SIAM Journal on Mathematical Analysis, 1993, 24(2):407- 435.

[8] Li M Y, Muldowney J S. Global Stability for the SEIR Model in Epidemiology[J]. Mathematical Biosciences, $1995,125(2): 155-164$.

[9] Cooke K L, Yorke J A. Some Equations Modelling Growth Processes and Gonorrhea Epidemics. Mathematical Biosciences, 1973, 16(1):75-101.

[10] Cooke K L, Van Den Driessche P. Analysis of an SEIRS Epidemic Model with Two Delays. Journal of Mathematical Biology, 1996, $35(2): 240-260$.

[11] Culshaw R V, Ruan S, Webb G. A Mathematical Model of Cell-to-Cell Spread of HIV-1 that Includes a Time Delay. Journal of Mathematical Biology, 2003, 46(5):425-444.

[12] Hethcote H W, Van Den Driessche P. Two SIS Epidemiologic Models with De- lays. Journal of Mathematical Biology, 2000, $40(1): 3-26$.

[13] Muroya Y, Enatsu Y, Nakata Y. Global Stability of a Delayed SIRS Epidemic Model with a Non-Monotonic Incidence Rate. Journal of Mathematical Analysis and Applications, 2011, 377(1):1-14.

[14] Rao, R. Stability Analysis of Nontrivial Stationary Solution and Constant Equilibrium Point of Reaction-Diffusion Neural Networks with Time Delays under Dirichlet Zero Boundary Value. Preprints 2020, 2020040277 (doi: 10.20944/preprints202004.0277.v6). 